\title{
Microwave-assisted facile green synthesis of silver nanoparticles and spectroscopic investigation of the catalytic activity
}

\author{
SIBY JOSEPH ${ }^{1, *}$ and BEENA MATHEW ${ }^{2}$ \\ ${ }^{1}$ Department of Chemistry, St. George's College, Aruvithura, Kottayam 686 122, Kerala, India \\ ${ }^{2}$ School of Chemical Sciences, Mahatma Gandhi University, Kottayam 686 560, Kerala, India
}

MS received 23 July 2014; revised 2 October 2014

\begin{abstract}
Silver nanoparticles have been successfully synthesized in aqueous medium by a green, rapid and costefficient synthetic approach based on microwave irradiation. In this study, iota-carrageenan (I-carrageenan) is used both as reducing and stabilizing agent. The formation of nanoparticles is determined using UV-vis, Fourier transform infrared (FTIR), X-ray diffraction (XRD), energy-dispersive X-ray (EDX) and high-resolution-transmission electron microscopic (HR-TEM) analysis. Transmission electron microscopic (TEM) images show that the nanoparticles are of spherical shape with an average diameter of $18.2 \mathrm{~nm}$. I-carrageenan-stabilized silver nanoparticles show outstanding catalytic activity for the reduction of 4-nitrophenol in the presence of $\mathrm{NaBH}_{4}$ in aqueous medium. The reaction follows pseudo-first-order kinetics and the reaction rate increases with the increase in amount of the catalyst. The study of the temperature dependence of reaction rate gives activation energy of $42.81 \mathrm{~kJ} \mathrm{~mol}^{-1}$. The synthesized silver nanoparticles are anticipated to be a promising material for pollution abatement.
\end{abstract}

Keywords. Microwave; green; silver nanoparticle; I-carrageenan; catalysis.

\section{Introduction}

Nanoscience and nanotechnology has emerged as one of the most active areas of research all over the globe in the last decades. This is due to the unique properties of nano-sized particles and because of their potential applications in various fields such as catalysis, ${ }^{1}$ optics ${ }^{2}$ and biomedical. ${ }^{3}$ Nanomaterials may provide solutions to many of our technological and environmental challenges. Among noble metal nanoparticles, silver has received considerable attention owing to its interesting physicochemical properties and the strong toxicity to a wide range of microorganisms. The chemical and physical properties of silver nanoparticles (AgNPs) are highly dependent on their size and shape, and these are strongly influenced by the experimental conditions. In recent years, a number of methods have been explored to synthesize silver nanostructures of suitable size and morphology. These include chemical reduction, ${ }^{4}$ polyol process, ${ }^{5}$ electrochemical, ${ }^{6}$ photochemical, ${ }^{7}$ sonochemical ${ }^{8}$ and biological methods. ${ }^{9}$ The synthesis and the application of AgNPs require the stabilization of them in suitable protecting systems. Systems such as polymers, ${ }^{10-12}$ hydrogels, ${ }^{13}$ microgels, ${ }^{14}$ dendrimers, ${ }^{15}$ etc. were used for this purpose. Among these, the most popular method for the preparation of $\mathrm{Ag}$ colloids is chemical reduction of silver salt in the presence of a stabilizing agent. The advantage of chemical method is that it takes short time for the synthesis of large

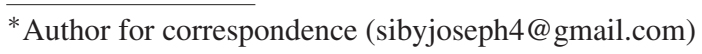

amount of nanoparticles. However, the chemical reagents used in this method as reducing and stabilizing agents are highly toxic and pose huge environmental hazards. Therefore, it is necessary to develop environmentally benign synthetic strategies for the preparation of AgNPs, especially in large scale.

Green synthesis is more advisable than chemical methods as it is cost effective, eco-friendly and thus can be easily scaled up for bulk synthesis. In green synthesis, three important rules of green chemistry should be considered including (i) selection of an environmentally benign solvent medium, (ii) selection of a non-toxic reducing agent and (iii) selection of a cost effective and easily renewable stabilizing agent for AgNPs. ${ }^{16}$ With the increase in focus on greener methods, natural compounds, ${ }^{17}$ plant extracts ${ }^{18}$ and microorganisms ${ }^{19}$ have aroused much interest as safer alternatives to chemical reducing and stabilizing agents. But one main drawback of biological methods is the longer reaction time. Microwave-assisted synthesis is particularly important in this regard. Microwave-assisted synthesis using biomaterials as both reducing and capping agents is a feasible way for the rapid and facile green synthesis of AgNPs. It provides several desirable features such as shorter reaction time, lower energy consumption and better product yield. Microwave irradiation offers rapid and uniform heating of the reaction medium and thus provides uniform nucleation and growth conditions for nanoparticles. Transfer of energy from the microwave radiation to the reactants is achieved through the interaction of radiation with water or other solvents with high dielectric constant or solvent molecules with 
large dipole moments. ${ }^{20}$ In recent years, many flourishing reports on microwave-assisted green synthesis of AgNPs have been published. ${ }^{21-24}$

Nitrophenols are considered to be one of the most hazardous and toxic unmanageable organic pollutants in waste waters generated from agricultural and industrial sources, including those manufacturing dyes, explosives, pesticides and other products. These pollutants are chemically stable and so traditional water treatment methods are usually ineffective. Therefore, developing an efficient and eco-friendly method for removing these pollutants from waste water is of immense importance from the view point of pollution abatement. In recent times, nanotechnology has been extended to the area of waste water treatment. Several groups reported the use of nanomaterials for the elimination of nitrophenols from water by reducing them into aminophenols which is an important intermediate in drug industry. ${ }^{25-30}$

In the present study, we report a facile green method for the synthesis of AgNPs in aqueous medium. Here, AgNPs are synthesized by microwave irradiation using iotacarrageenan (I-carrageenan) as both reducing and stabilizing agent. I-carrageenans are sulphated linear polysaccharides extracted from certain red edible seaweeds. The structure is built up of alternating 1,3-linked $\beta$-D-galactopyranosyl and 1,4-linked $\alpha$-D-galactopyranosyl units. I-carrageenan has two sulphates per disaccharide. The presence of hydroxyl and sulphate groups in carrageenan structure makes them likely to be hydrophilic. Hydrogels produced from I-carrageenan are soft, flexible and fast setting. They have been extensively used in the food industry as a thickening and gelling agent. In recent times, they are used as excepient in pills and tablets. Because I-carrageenan is an easily renewable naturally occurring biopolymer, this method offers a simple, environmentally friendly and cost-effective method for the synthesis of AgNPs. The catalytic efficiency of the synthesized nanoparticles was investigated using the reduction of 4-nitrophenol to 4-aminophenol by $\mathrm{NaBH}_{4}$.

\section{Materials and methods}

\subsection{Materials}

Silver nitrate $\left(\mathrm{AgNO}_{3}\right)$, 4-nitrophenol and sodium borohydride $\left(\mathrm{NaBH}_{4}\right)$ of analytical grade were purchased from Merck India Ltd. I-carrageenan was obtained from Himedia Chemicals (Mumbai, India). Double distilled water was used for all experiments.

\subsection{Methods}

2.2a Synthesis of AgNP-carrageenan: In a typical synthesis, $0.1 \mathrm{~g}$ of carrageenan was dissolved in $90 \mathrm{ml}$ of hot water in a beaker. To this, $10 \mathrm{ml}$ of $0.05 \mathrm{M} \mathrm{AgNO}_{3}$ solution was added so as to keep the concentration of $\mathrm{AgNO}_{3}$ in the reaction mixture as $0.005 \mathrm{M}$ and the solution was stirred for $15 \mathrm{~min}$. The reaction mixture was then placed in a domestic microwave oven (Sharp R-219T (W)) operating at a power of $800 \mathrm{~W}$ and frequency $2450 \mathrm{MHz}$ and was subjected to microwave irradiation for $2 \mathrm{~min}$. Upon microwave irradiation, the colourless solution turned into dark brown, indicating the reduction of silver ions into silver colloids. The formation of AgNPs was monitored using UV-vis spectrophotometer by analysing the reaction mixture after different irradiation times.

2.2b Reduction of 4-nitrophenol to 4-aminophenol: The reduction of 4-nitrophenol to 4-aminophenol by $\mathrm{NaBH}_{4}$ was used to study the catalytic activity of AgNP-carrageenan. To follow this reaction, $2 \mathrm{ml}$ of 4-nitrophenol $\left(0.08 \times 10^{-3} \mathrm{M}\right)$ was taken in a quartz cuvette of $1 \mathrm{~cm}$ path length and to this $0.5 \mathrm{ml}$ freshly prepared $\mathrm{NaBH}_{4}$ solution $(0.06 \mathrm{M})$ was added. Then $0.5 \mathrm{ml}$ of AgNP-carrageenan hydrosol was added to start the reaction. The reaction was monitored by analysing the absorption peak at $400 \mathrm{~nm}$ using UV-vis spectrophotometer. The absorption spectra were taken in 1 min intervals in the range of $200-600 \mathrm{~nm}$ at $24^{\circ} \mathrm{C}$.

2.2c Characterization: The UV-vis spectra were recorded on a Shimadzu UV-2450 spectrophotometer. Fourier transform infrared (FTIR) spectra were measured using Perkin-Elmer-400 spectrometer with ATR facility. Energydispersive X-ray (EDX) measurement was carried out using JEOL JSM-6390 scanning electron microscope (SEM) with EDX attachment. X-ray diffraction (XRD) measurements were carried out using PANalytic XPERT-PRO X-ray spectrometer. High-resolution transmission electron microscopic (HR-TEM) images were obtained using a JEOL JEM-2100 microscope.

\section{Results and discussion}

\subsection{Synthesis of AgNPs}

The evidence for the successful formation of carrageenanstabilized AgNPs (AgNP-carrageenan) is obtained from UV-vis absorption spectral studies. The UV-vis absorption spectra of the solution recorded at $1 \mathrm{~min}$ intervals during microwave irradiation process are shown in figure 1 . No obvious absorption is observed in the range of $250-600 \mathrm{~nm}$ for I-carrageenan. After microwave irradiation for $1 \mathrm{~min}$, an absorption band was observed at about $416 \mathrm{~nm}$ and the intensity of which became stronger with passage of reaction time. This prominent absorption band is attributed to the characteristic surface plasmon resonance (SPR) absorption of silver nanoparticles. Also, there is no obvious absorption in the range of $450-800 \mathrm{~nm}$, which indicates that negligible aggregation occurs in this reaction system and the nanoparticles are well dispersed. ${ }^{31}$ Initially when $\mathrm{AgNO}_{3}$ was added to carrageenan solution, the solution remained colourless. But upon microwave irradiation, the colourless solution gradually changes to dark brown. This colour difference also indicates the formation of AgNPs. The silver 


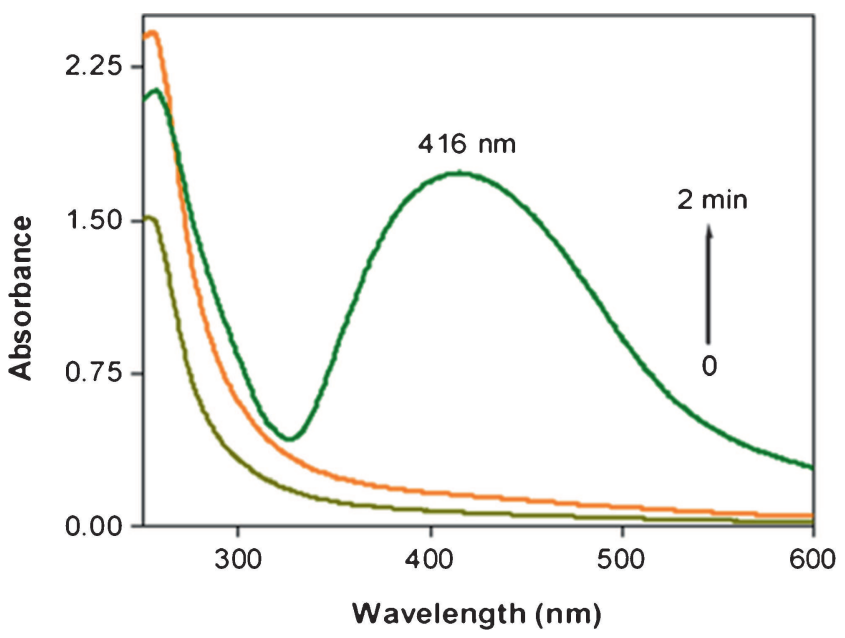

Figure 1. UV-vis absorption spectra of AgNP-carrageenan at different microwave irradiation times.

colloids are found to be stable for 3 months due to efficient capping by I-carrageenan molecules. These results indicate that I-carrageenan is able to reduce $\mathrm{Ag}^{+}$ions into $\mathrm{Ag}$ nanoparticles and it is also a good stabilizer for the generated AgNPs. When $\mathrm{AgNO}_{3}$ solution is added to I-carrageenan solution, $\mathrm{Ag}^{+}$ions get entrapped within the carrageenan chain because the polar- $\mathrm{OH}$ groups and electron-rich oxygen atoms on the carrageenan chain act as templates for the incoming $\mathrm{Ag}^{+}$ions. The sulphate groups of carrageenan efficiently reduce the $\mathrm{Ag}^{+}$ions into $\mathrm{Ag}$ under the influence of microwave radiation which combines to form highly dispersed AgNPs. The problem of inhomogeneous heating with conventional thermal methods is overcome in microwave method. The use of microwave radiation provides increased reaction kinetics, rapid initial heating and hence enhanced reaction rates with rapid consumption of starting materials. ${ }^{32}$ This will prevent agglomeration of nanoparticles to a greater extent resulting in monodispersed AgNPs. Thus microwave irradiation is an effective and economically viable heating method for the production of nanomaterials.

\subsection{FTIR spectral studies}

The FTIR spectrum of I-carrageenan (figure 2) shows a broad band at $3200-3400 \mathrm{~cm}^{-1}$. This is due to stretching vibrations of $\mathrm{OH}$ groups of I-carrageenan. The peak observed at $2930 \mathrm{~cm}^{-1}$ is attributed to the $\mathrm{C}-\mathrm{H}$ stretching vibrations of $\mathrm{CH}_{2}$ groups. A strong band observed at $1386 \mathrm{~cm}^{-1}$ is characteristic of asymmetric stretching vibrations of $\mathrm{S}=\mathrm{O}$ group of sulphate esters. The strong band at $1023 \mathrm{~cm}^{-1}$ is ascribed to stretching vibrations of $\mathrm{C}-\mathrm{O}-\mathrm{C}$ glycosidic linkage and that at $843 \mathrm{~cm}^{-1}$ is due to $\mathrm{C}-\mathrm{O}-\mathrm{S}$ vibrations of D-galactose4-sulphate. The spectrum of AgNP-carrageenan is almost similar to that of pristine I-carrageenan. However one can see some minor shifts in the various stretching frequencies especially that for the peaks due to $\mathrm{S}=\mathrm{O}$ group and $\mathrm{C}-\mathrm{O}-$ $\mathrm{C}$ glycosidic linkage. These observations afford an obvious

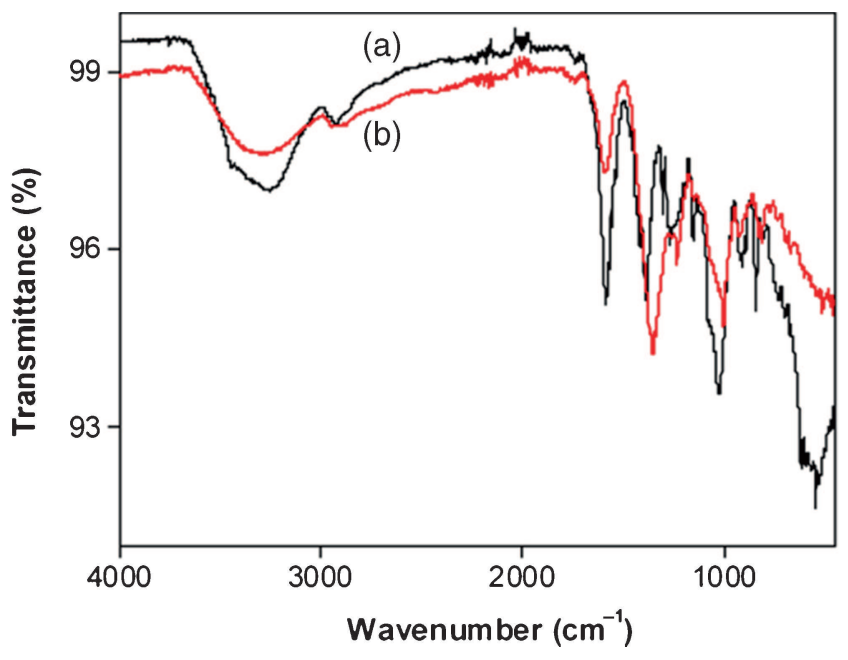

Figure 2. FTIR spectra of (a) I-carrageenan and (b) AgNPcarrageenan.

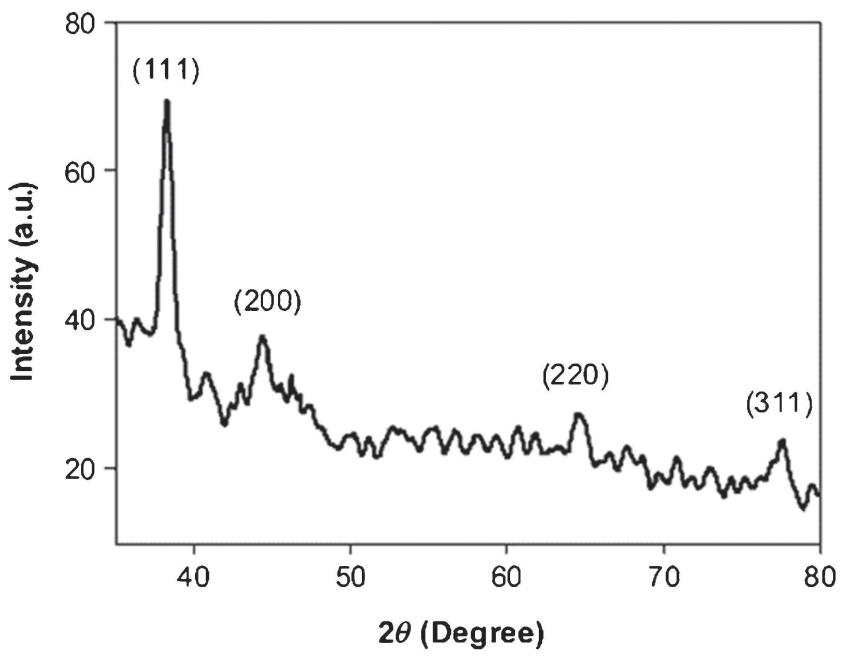

Figure 3. XRD pattern of AgNP-carrageenan.

indication for the formation and stabilization of AgNPs by I-carrageenan.

\subsection{XRD pattern}

XRD study was performed to confirm the crystalline nature of AgNPs. In figure 3, four distinct XRD peaks are clearly observed at $2 \theta$ values of $38.30^{\circ}, 44.32^{\circ}, 64.56^{\circ}$ and $77.53^{\circ}$. These peaks are indexed, respectively, to the reflections of the (111), (200), (220) and (311) crystallographic planes of face centred cubic (fcc) silver particles. All diffraction peaks are in good agreement with the standard values (JCPDS file no. 04-0783). The intensity of the peak due to reflections of (111) plane is relatively higher than that of other planes. This indicates that the AgNPs are enriched in (111) facets and the (111) plane appears to be preferentially oriented parallel to the surface of the supporting material. 


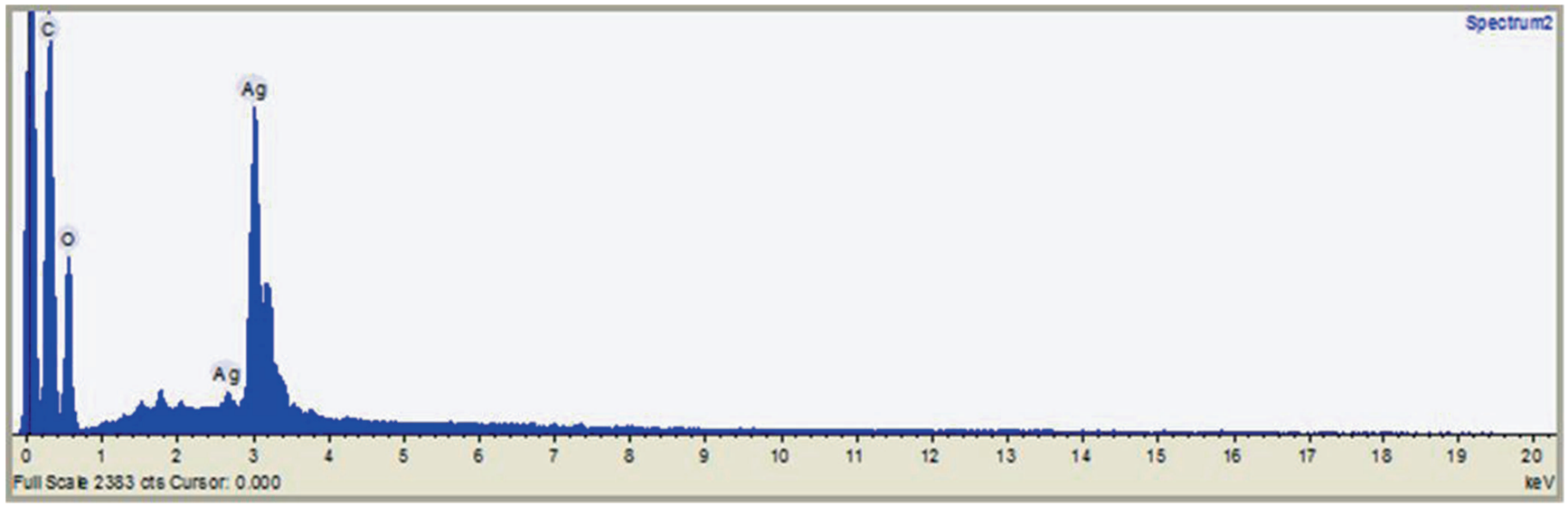

Figure 4. EDX spectrum of AgNP-carrageenan.
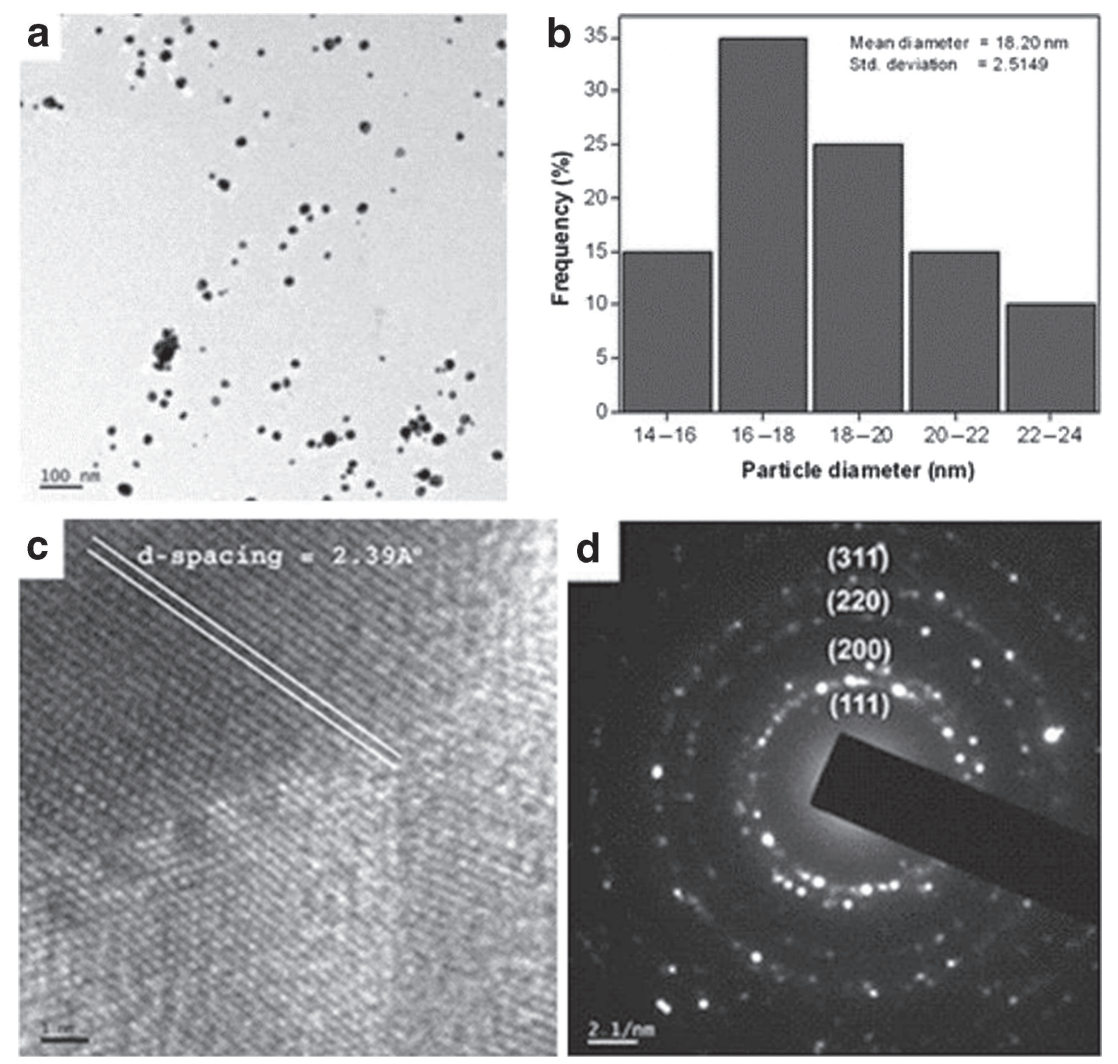

Figure 5. (a) TEM image, (b) the particle size histogram, (c) HR-TEM image and (d) the selected area electron diffraction (SAED) pattern of AgNP-carrageenan.

\subsection{EDX analysis}

The elemental characterization of the synthesized AgNPs was conducted by EDX analysis. The peaks located between 2.6 and $3.6 \mathrm{keV}$ in the EDX spectrum of AgNP-carrageenan (figure 4) are directly related to the characteristic $\mathrm{K}$ and $\mathrm{L}$ lines of silver. The maximum observed at about $0.3 \mathrm{keV}$ is characteristic of carbon. The peak located at about $0.5 \mathrm{keV}$ is related with oxygen. The carbon and oxygen signals observed in the spectrum of the sample originate from carrageenan. This confirms the stabilization of AgNPs by carrageenan chains.

\subsection{HR-TEM analysis}

The size and morphology of the synthesized AgNPs were investigated using HR-TEM analysis. The TEM images of AgNP-carrageenan are given in figure 5. As is clear from figure $5 \mathrm{a}$, the nanoparticles are more or less uniformly distributed and they are almost spherical in shape. They are 


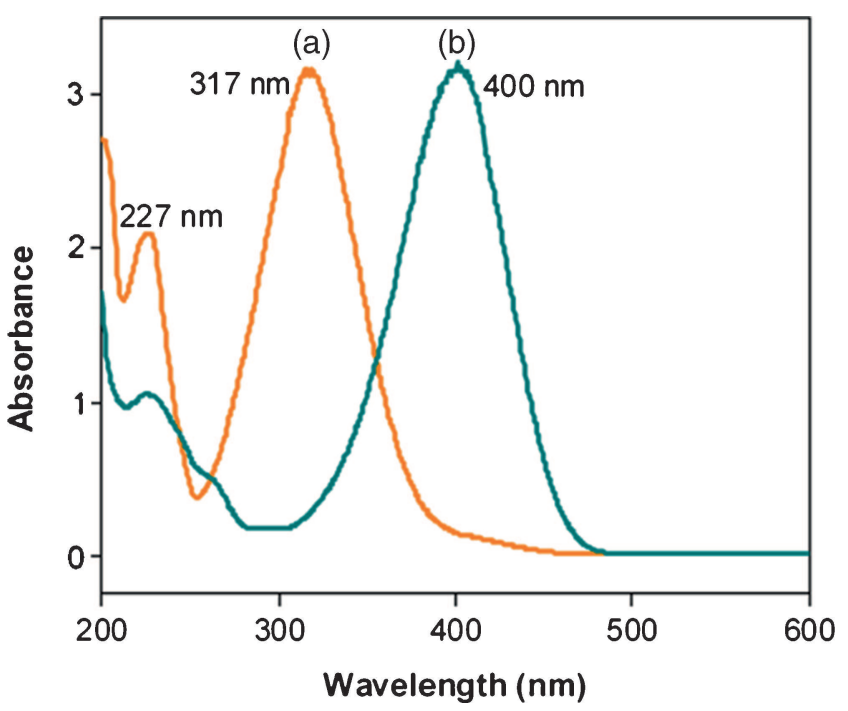

Figure 6. UV-vis absorption spectrum of (a) 4-nitrophenol and (b) 4-nitrophenolate ion.

also highly crystalline. The particle size histogram drawn using the data obtained from the TEM image is shown in figure 5b. The average particle size is observed as $18.2 \mathrm{~nm} \pm 2$. As can be seen, nearly $60 \%$ of the particles have diameter between 16 and $20 \mathrm{~nm}$ and the size distribution plot appears to be almost symmetrical with all the nanoparticles coming within the range of 14-24 nm. The HR-TEM image (figure 5c) shows clear lattice fringes. The fringe spacing is measured as $2.39 \mathrm{~A}^{\circ}$. This spacing corresponds to that between (111) planes of fcc silver (JCPDS file no. 040783). This also reveals that the crystal growth of nanoparticles occurred mainly on the (111) plane. The selected area electron diffraction (SAED) pattern is shown in figure $5 \mathrm{~d}$. The diffraction rings are visible clearly and these can be indexed to the (111), (200), (220) and (311) reflections of fcc AgNPs. All these results give a clear indication of the highly crystalline nature of the AgNPs synthesized by this method.

\subsection{Catalytic reduction of 4-nitrophenol to 4-aminophenol}

To investigate the competence of AgNP-carrageenan as a catalyst, the reduction of 4-nitrophenol by $\mathrm{NaBH}_{4}$ was selected as a model reaction. The UV-vis absorption spectrum of 4-nitrophenol has maximum absorption at $317 \mathrm{~nm}$. When $\mathrm{NaBH}_{4}$ was added to the aqueous solution of 4-nitrophenol, the yellow colour of the solution became more intense and the peak due to 4-NP was shifted to $400 \mathrm{~nm}$ (figure 6). This is due to the formation of 4-nitrophenolate ions under the alkaline condition created on the addition of $\mathrm{NaBH}_{4}{ }^{33}$

The intensity of the peak at $400 \mathrm{~nm}$ due to 4-nitrophenolate ion remained almost unchanged even after several hours indicating that $\mathrm{NaBH}_{4}$ alone is unable to effectively reduce 4nitrophenol (4-NP) to 4-aminophenol (4-AP). But the reduction reaction started immediately upon the addition of a small amount of AgNP-carrageenan catalyst. This is evident from

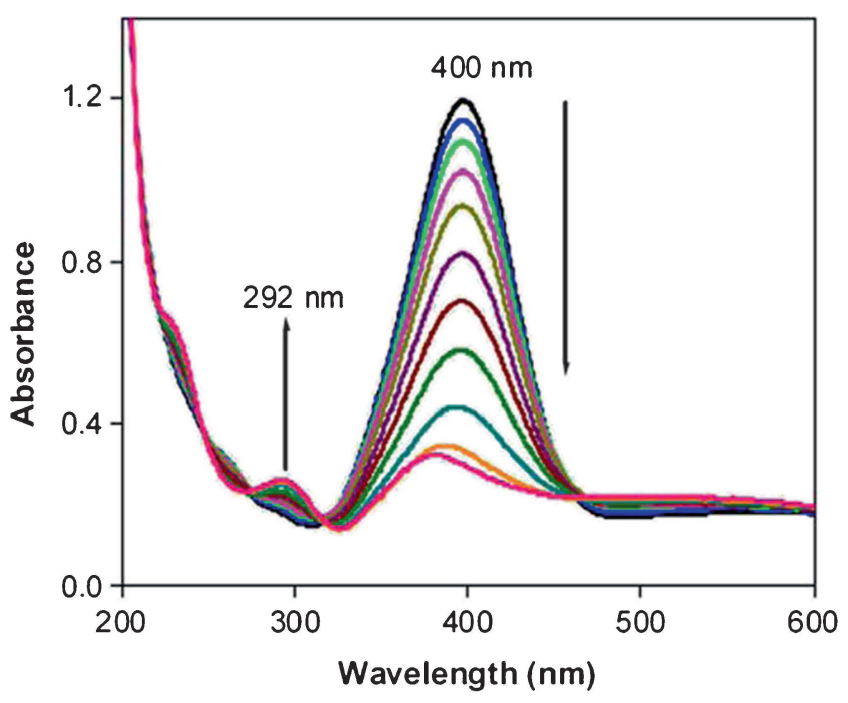

Figure 7. Successive UV-vis absorption spectra for the $\mathrm{NaBH}_{4}$ reduction of 4-nitrophenol recorded at $1 \mathrm{~min}$ intervals catalysed by AgNP-carrageenan at $24^{\circ} \mathrm{C}$. Conditions: $[4-\mathrm{NP}]=0.08 \times 10^{-3} \mathrm{M}$, $\left[\mathrm{NaBH}_{4}\right]=0.06 \mathrm{M},[$ AgNP-carrageenan $]=0.03 \mathrm{mg} \mathrm{ml}^{-1}$.

the fading and ultimate bleaching of the yellow colour of the reaction mixture. The $\mathrm{UV}-$ vis absorption spectra for the $\mathrm{NaBH}_{4}$ reduction of $4-\mathrm{NP}$ at $24^{\circ} \mathrm{C}$ catalysed by $0.03 \mathrm{mg} \mathrm{ml}^{-1}$ AgNP-carrageenan is shown in figure 7 . The spectrum was recorded every $1 \mathrm{~min}$ after the addition of the catalyst to the reaction medium in order to follow the progress of the reaction.

It is evident from the figure that, after the addition of AgNP-carrageenan catalyst, the intensity of the peak at 400 nm decreases continuously with time and a new peak appears at $292 \mathrm{~nm}$ whose intensity increases with elapse of time. This new peak is due to the formation of the product 4aminophenol (equation (1)). The reaction is found to be complete in $9 \mathrm{~min}$.

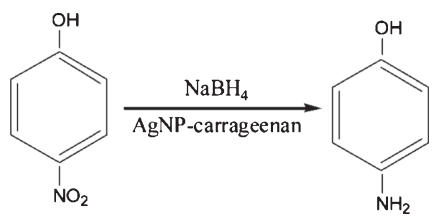

As reaction progresses, not only a decrease in the intensity of the peak at $400 \mathrm{~nm}$ but also a blue shift of the peak from 400 to $382 \mathrm{~nm}$ was observed. The peak at $382 \mathrm{~nm}$ is not that of 4-nitrophenol. This peak is due to the surface plasmon resonance (SPR) of silver hydrosol. In the intermediate stage of the reaction, the SPR band of AgNPs is not observed because it remains masked under the absorption band of 4-nitrophenolate ion. But finally, this band become visible when all the reactants are converted to 4-AP. The SPR band of AgNP-carrageenan is actually at $416 \mathrm{~nm}$. When AgNP-carrageenan is added as catalyst to the reaction mixture containing 4-NP and excess of $\mathrm{NaBH}_{4}$, the borohydride ions inject electrons on to the catalyst surface. This leads to 
a blue shift in the SPR band of AgNP-carrageenan from 416 to $382 \mathrm{~nm} .^{34}$

The mechanism of this reduction reaction involves the initial adsorption of both 4-nitrophenolate ions and $\mathrm{BH}_{4}^{-}$ions on the catalyst surface. Because of the close proximity of the reacting groups on the catalyst surface, the reduction reaction becomes very easy. The catalyst helps this reduction process by relaying electrons from the donor $\mathrm{BH}_{4}^{-}$ions to the acceptor 4-nitrophenolate ions.

The kinetics of this reaction can be followed quantitatively by monitoring the change in the intensity of the peak at 400 $\mathrm{nm}$ with time. In this study, the concentration of $\mathrm{NaBH}_{4}$ largely exceeds that of 4-NP. As the initial concentration of $\mathrm{NaBH}_{4}$ is very high, it remains essentially constant during the course of the reaction. Hence pseudo-first-order kinetics with respect to 4-nitrophenol could be used to evaluate the reaction rate. The kinetics of the reaction can be represented using the equation $\ln [A] /\left[A_{\circ}\right]=-k t$, where $k$ is the firstorder rate constant, $t$ is the reaction time, $\left[A_{\circ}\right]$ is the concentration of 4-NP at time $t=0$ and $[A]$ is the concentration at time $t$. The value of $[A]$ can be obtained from the absorbance of the peak at $400 \mathrm{~nm}$.

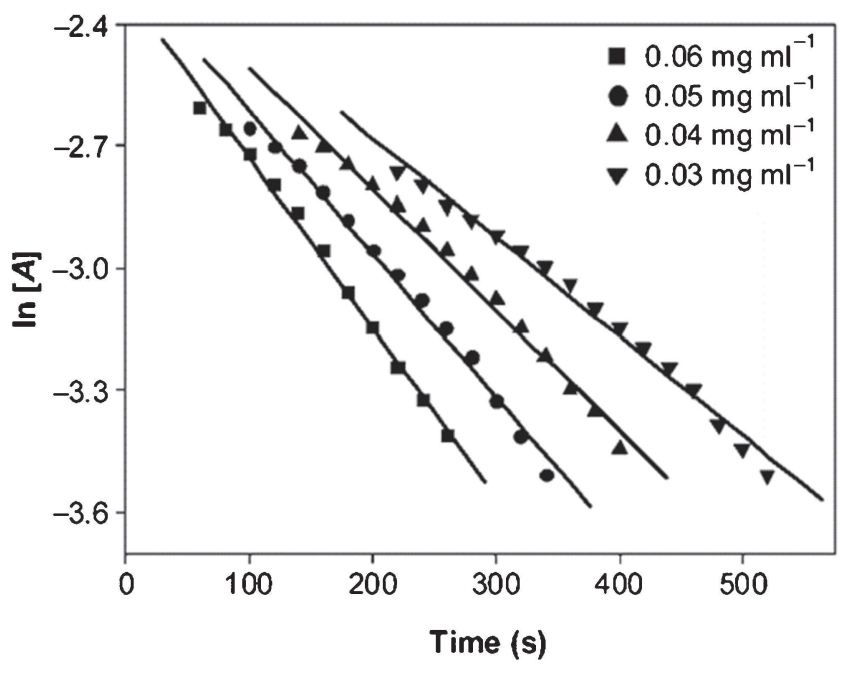

Figure 8. Plot of $\ln [A]$ against time for the reduction of 4-nitrophenol using varying amount of AgNP-carrageenan catalyst. Conditions: [4$\mathrm{NP}]=0.08 \times 10^{-3} \mathrm{M},\left[\mathrm{NaBH}_{4}\right]=0.06 \mathrm{M}$, temperature $=24^{\circ} \mathrm{C}$.

Table 1. Pseudo-first-order rate constants for the reduction of 4-NP using varying amount of AgNP-carrageenan catalyst.

\begin{tabular}{lccc}
\hline $\begin{array}{l}\text { AgNP-carrageenan } \\
\left(\mathrm{mg} \mathrm{ml}^{-1}\right)\end{array}$ & $\begin{array}{c}\text { Reaction time } \\
(\mathrm{min})\end{array}$ & $\begin{array}{c}\mathrm{k} \times 10^{-3} \\
\left(\mathrm{~s}^{-1}\right)\end{array}$ & $\begin{array}{c}\text { Correlation } \\
\text { coefficient }\left(R^{2}\right)\end{array}$ \\
\hline 0.030 & 9 & 2.43 & 0.9882 \\
0.040 & 7 & 2.98 & 0.9904 \\
0.050 & 6 & 3.51 & 0.9905 \\
0.060 & 5 & 4.17 & 0.9944 \\
\hline
\end{tabular}

To study the effect of amount of the catalyst upon rate of the reduction reaction, the reaction was conducted by varying the concentration of AgNP-carrageenan hydrosol in the reaction mixture, keeping other parameters constant. The reaction rate was analysed by following the decrease in the absorbance value at $400 \mathrm{~nm}$ with time. As shown in figure 8 , a linear relation between $\ln [A]$ and time has been observed in all catalytic reactions studied. This confirms the pseudofirst-order nature of the reaction. The first-order rate constant $k$ for these reactions is obtained from the slope of the linear plots.

The first-order rate constant obtained from the slope of $\ln [A] v s$. time plot for different amounts of catalyst and the corresponding correlation coefficients are given in table 1 .

It is clear from the table that, these reactions are very fast and strictly follows pseudo-first-order kinetics. Also, the values of the rate constant obtained for different concentrations of the catalyst are plotted in figure 9 . We can see that, the rate constant increases with the increase in the concentration of the catalyst. Moreover, a strictly linear relation between $k$ and the concentration of catalyst can be observed. The reaction time decreases with the increase in the amount of catalyst. The absorbance value of the peak observed at $389 \mathrm{~nm}$ after complete reduction of 4-NP due to surface plasmon resonance of nano-silver increases with the increase in catalyst concentration. This is due to the presence of more amount of catalyst in the reaction medium after the reduction process.

Furthermore, initially an induction time (IT), the time taken to observe any appreciable change in the absorbance at $400 \mathrm{~nm}$ was found for all the catalytic reaction when carried out under air and after that the rate increases gradually with time. This observation has been made by several other research groups. ${ }^{7,28,34,35}$ It was also found that the induction time in nitrogen atmosphere is less and negligible in comparison to that in ambient condition. ${ }^{34}$ This is because of the surface oxidation of the catalyst in oxygen atmosphere

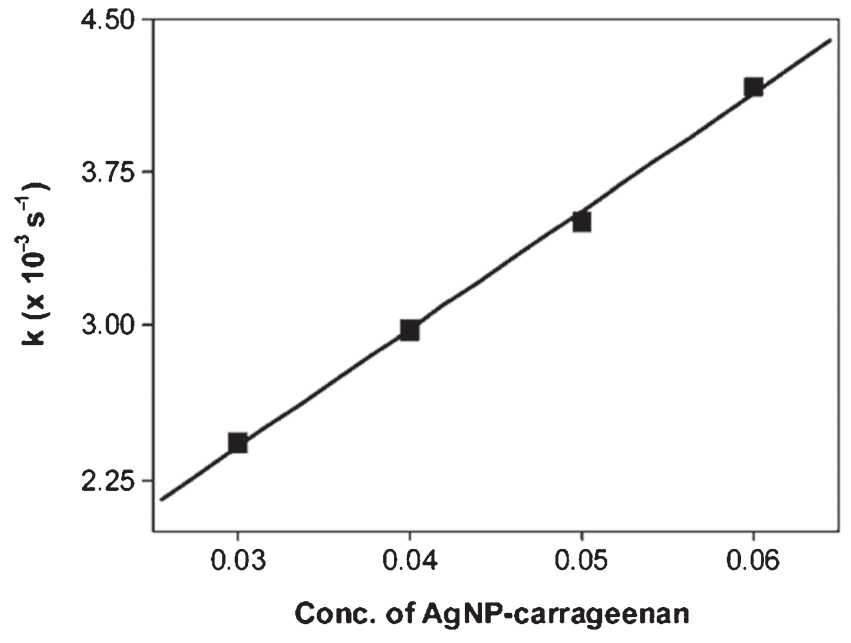

Figure 9. Plot of rate constant against amount of catalyst for the reduction of 4-NP. Conditions: $[4-\mathrm{NP}]=0.08 \times 10^{-3} \mathrm{M}$, $\left[\mathrm{NaBH}_{4}\right]=0.06 \mathrm{M}$, temperature $=24^{\circ} \mathrm{C}$. 
resulting in the formation of oxide layer on the catalyst surface which may poison the catalyst. $\mathrm{NaBH}_{4}$ reduces the oxide layer and regenerate fresh catalyst surface for the adsorption of reactants. Another reason for the IT is the time taken for the reduction of $\mathrm{O}_{2}$ by $\mathrm{NaBH}_{4} \cdot{ }^{36}$ The reduction of 4-NP starts only after $\mathrm{O}_{2}$ is removed from the reaction medium by reduction. Because $\mathrm{NaBH}_{4}$ is taken in large excess, its consumption for the above processes does not alter its concentration appreciably. Moreover, the hydrogen liberated during the reduction process scavenges $\mathrm{O}_{2}$ and thus prevent the oxidation of the reduction product as well as the catalyst. IT is observed to decrease with increase in the catalyst concentration as well as temperature.

To study the effect of temperature on the rate of AgNPcarrageenan catalysed reduction of 4-nitrophenol, the reaction was carried out at four different temperatures $(24,26$, 28 and $30^{\circ} \mathrm{C}$ ) and the pseudo-first-order rate constant was calculated in each case. The activation energy $\left(E_{\mathrm{a}}\right)$ for the reaction can be calculated using the well-known Arrhenius equation

$$
\ln k=-E_{\mathrm{a}} / R T+\ln A .
$$

In this equation, $k$ is the rate constant, $R$ the gas constant ( $8.314 \mathrm{~J} \mathrm{~K}^{-1} \mathrm{~mol}^{-1}$ ), $T$ the absolute temperature and $A$ the Arrhenius parameter. From equation (2), it follows that the plot of $\ln k$ vs. $1 / T$ should yield a straight line, the slope of which is equal to $-E_{\mathrm{a}} / R$. From this, $E_{\mathrm{a}}$ can be evaluated. The values of $\ln k$ obtained in our study at different temperatures are plotted against $1 / T$ in figure 10 . As is clear, the value of $k$ increases with the increase in temperature showing that the reaction is accelerated by increasing temperature.

From the Arrhenius plot, the activation energy for the reaction is calculated to be $42.81 \mathrm{~kJ} \mathrm{~mol}^{-1}$. Pradhan et $a l^{34}$ studied the AgNP catalysed reduction of 4-nitrophenol and reported the activation energy of $41 \mathrm{~kJ} \mathrm{~mol}^{-1}$. Shin et al ${ }^{36}$

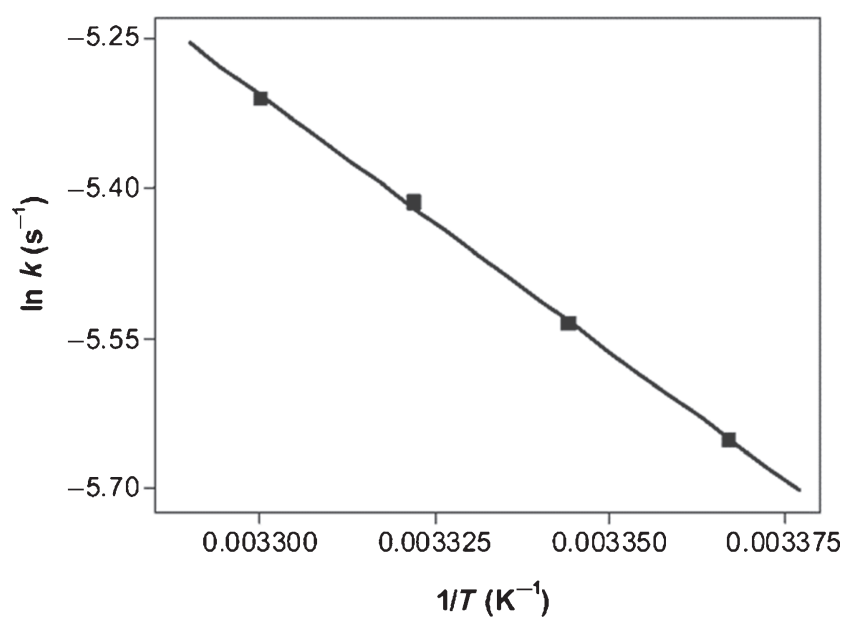

Figure 10. Arrhenius plot of $\ln k$ against $1 / T$ for the reduction of 4-nitrophenol catalysed by AgNP-carrageenan. Conditions: $[4-\mathrm{NP}]=0.08 \times 10^{-3} \mathrm{M},\left[\mathrm{NaBH}_{4}\right]=0.06 \mathrm{M},[$ AgNP-carrageenan $]$ $=0.05 \mathrm{mg} \mathrm{ml}^{-1}$. also studied the same reaction using silver-deposited magnetic nanoparticles and the activation energy was found to be $45 \mathrm{~kJ} \mathrm{~mol}^{-1}$. However, Sahiner and group ${ }^{37}$ conducted the reduction of 4-NP using polyacrylamidoglycolic acid-silver composite and reported the activation energy of $33.8 \mathrm{~kJ} \mathrm{~mol}^{-1}$. The result obtained in our study is in good agreement with the results of Pradhan et al. ${ }^{34}$

\section{Conclusions}

In this study, we have reported a rapid facile microwave based method for the green synthesis of AgNPs in aqueous medium which are stable for a long period. This method uses the easily renewable biopolymer I-carrageenan as both reducing and capping agent. The various characteristics of the synthesized silver colloids are analysed by UV-vis, FTIR, XRD, EDX and TEM analyses. The catalytic activity is scrutinized using the reduction of 4-nitrophenol to 4-aminophenol by $\mathrm{NaBH}_{4}$ as a model reaction. The reaction follows pseudo-first-order kinetics and the rate constant is found to increase as the concentration of the catalyst increases. The temperature dependence of reaction rate is studied by conducting the reaction at four different temperatures. The rate of reaction is found to increase with the increase in temperature and the activation energy is found to be $42.81 \mathrm{~kJ} \mathrm{~mol}^{-1}$. Since this method for the synthesis of nanoparticles is relatively simple, fast and economic, this has the potential for the bulk production of silver colloids and their industrial application as a catalyst for the reduction of toxic nitrocompounds.

\section{Acknowledgement}

This work was supported by UGC Bangalore, Government of India, under FIP scheme.

\section{References}

1. Esumi K, Miyamoto K and Yoshimura T 2002 J. Colloid Interface Sci. 254402

2. Eychmuller A 2000 J. Phys. Chem. B 1046514

3. Salata O V 2004 J. Nanobiotechnol. 23

4. Pastoriza-Santos I and Liz-Marzan L M 2002 Langmuir 18 2888

5. Sun Y and Xia Y 2002 Adv. Mater. 14833

6. Yin B, Ma H, Wang S and Chen S 2003 J. Phys. Chem. B 107 8898

7. Kundu Mandal M, Ghosh S K and Pal T 2004 J. Colloid Interface Sci. 272134

8. Okitsu K, Yue A, Tanabe S and Matsumoto H 2000 Chem. Mater. 123006

9. Shankar S S, Rai A, Ahmad A and Sastry M 2004 J. Colloid Interface Sci. 275496

10. Debnath D, Kim C and Kim S H 2010 Macromol. Rapid Commun. 31549 
11. Luo C, Zhang Y, Zeng X, Zeng Y and Wang Y 2005 J. Colloid Interface Sci. $\mathbf{2 8 8} 444$

12. Rastogi P K, Ganesan V and Krishnamoorthi S 2012 Mater. Sci. Eng. B 177456

13. Bajpai S K, Bajpai M and Gautam D 2013 J. Macromol. Sci.: Pure Appl. Chem. $\mathbf{5 0} 46$

14. Khan A, El-Toni A M, Alrokayan S, Alsalhi M, Alhoshan M and Aldwayyan A S 2011 Colloids Surf. A 377356

15. Esumi K, Isono R and Yoshimura T 2004 Langmuir 20237

16. Raveendran P, Fu J and Wallen S L 2003 J. Am. Chem. Soc. 12513940

17. Vigneshwaran N, Nachane R P, Balasubramanya R H and Varadarajan P V 2006 Carbohydr. Res. 3412012

18. Vidhu V K, Aromal S A and Philip D 2011 Spectrochim. Acta A 83392

19. Vigneshwaran N, Ashtaputre N M, Varadarajan P V, Nachane R P, Par-Alikar K M and Balasubramanya R H 2007 Mater. Lett. 611413

20. Galema S A 1997 Chem. Soc. Rev. 26233

21. Sreeram K J, Nidhin M and Nair B U 2008 Bull. Mater. Sci. 31 937

22. Hu B, Wang S, Wang K, Zhang M and Yu S 2008 J. Phys. Chem. C 11211169
23. Peng H, Yang A and Xiong J 2013 Carbohydr. Polym. 91348

24. Siby J and Beena M 2014 J. Mol. Liquids 197346

25. Ghosh S K, Mandal M, Kundu S, Nath S and Pal T 2004 Appl. Catal. A 26861

26. Saha S, Pal A, Kundu S, Basu S and Pal T 2010 Langmuir 26 2885

27. Wunder S, Polzer F, Lu Y, Mei Y and Ballauff M 2010 J. Phys. Chem. C 1148814

28. Wei D, Ye Y, Jia X, Yuan C and Qian W 2010 Carbohydr. Res. 34574

29. Sen I K, Maity K and Islam S S 2013 Carbohydr. Polym. 91518

30. Nemanashi M and Meijboom R 2013 J. Colloid Interface Sci. 389260

31. Gutierrez M and Henglein A 1993 J. Phys. Chem. B 9711368

32. Nadagouda M N, Speth T F and Varma R S 2011 Acc. Chem. Res. 44469

33. Pradhan N, Pal A and Pal T 2001 Langmuir 171800

34. Pradhan N, Pal A and Pal T 2002 Colloids Surf. A 196247

35. Lu Y, Mei Y, Walker R, Ballauff M and Drechsler M 2006 Polymer 474985

36. Shin K S, Choi J Y, Park C S, Jang H J and Kim K 2009 Catal. Lett. 1331

37. Butun S and Sahiner N 2011 Polymer 524834 